TRANSACTIONS OF THE

AMERICAN MATHEMATICAL SOCIETY

Volume 364, Number 4, April 2012, Pages 2213-2226

S 0002-9947(2011)05499-1

Article electronically published on October 21, 2011

\title{
TOPOLOGICAL OBSTRUCTIONS TO TOTALLY SKEW EMBEDDINGS
}

\author{
ĐORĐE BARALIĆ, BRANISLAV PRVULOVIĆ, GORDANA STOJANOVIĆ, \\ SINIŠA VREĆICA, AND RADE ŽIVALJEVIĆ
}

\begin{abstract}
Following Ghomi and Tabachnikov's 2008 work, we study the invariant $N\left(M^{n}\right)$ defined as the smallest dimension $N$ such that there exists a totally skew embedding of a smooth manifold $M^{n}$ in $\mathbb{R}^{N}$. This problem is naturally related to the question of estimating the geometric dimension of the stable normal bundle of the configuration space $F_{2}\left(M^{n}\right)$ of ordered pairs of distinct points in $M^{n}$. We demonstrate that in a number of interesting cases the lower bounds on $N\left(M^{n}\right)$ obtained by this method are quite accurate and very close to the best known general upper bound $N\left(M^{n}\right) \leq 4 n+1$ established by Ghomi and Tabachnikov. We also provide some evidence for the conjecture that for every $n$-dimensional, compact smooth manifold $M^{n}(n>1)$,
\end{abstract}

$$
N\left(M^{n}\right) \leq 4 n-2 \alpha(n)+1 .
$$

\section{INTRODUCTION}

Two lines in an affine space $\mathbb{R}^{N}$ are called skew if they are neither parallel nor have a point in common or equivalently if their affine span has dimension 3. More generally, affine subspaces $U_{1}, \ldots, U_{l}$ of $\mathbb{R}^{N}$ are called skew if their affine span has dimension $\operatorname{dim}\left(U_{1}\right)+\cdots+\operatorname{dim}\left(U_{l}\right)+l-1$, in particular a pair $U, V$ of affine subspaces of $\mathbb{R}^{N}$ is skew if and only if each two lines $p \subset U$ and $q \subset V$ are skew.

An embedding $f: M^{n} \rightarrow \mathbb{R}^{N}$ of a smooth manifold is called totally skew if for each two distinct points $x, y \in M^{n}$ the affine subspaces $d f\left(T_{x} M\right)$ and $d f\left(T_{y} M\right)$ of $\mathbb{R}^{N}$ are skew. Define $N\left(M^{n}\right)$ as the minimum $N$ such that there exists a totally skew embedding of $M^{n}$ into $\mathbb{R}^{N}$.

In 3] Ghomi and Tabachnikov began the study of totally skew embeddings of manifolds and established a surprising connection of $N\left(M^{n}\right)$ with some classical invariants of geometry and topology. For example they showed [3, Theorem 1.4] that the problem of estimating $N\left(\mathbb{R}^{n}\right)$ is intimately related to the generalized vector field problem and the immersion problem for real projective spaces, as exemplified by the inequality

$$
N\left(\mathbb{R}^{n}\right) \geq r(n)+n,
$$

where $r(n)$ is the minimum $r$ such that the Whitney sum $r \xi_{n-1}$ of $r$ copies of the canonical line bundle over $\mathbb{R} P^{n-1}$ admits $n+1$ linearly independent continuous cross sections.

Received by the editors June 30, 2010 and, in revised form, October 18, 2010.

2010 Mathematics Subject Classification. Primary 57R40; Secondary 55R40, 57R20.

This research was supported by the Grants 174020 and 174034 of the Ministry for Science and Technological Development of Serbia.

(C)2011 American Mathematical Society Reverts to public domain 28 years from publication 
Another example ([3, Theorem 1.2]) is the inequality

$$
N\left(S^{n}\right) \leq n+m(n)+1,
$$

where $m(n)$ is an equally well-known function defined as the minimum $m$ such that there exists a non-singular, symmetric bilinear form $B: \mathbb{R}^{n+1} \times \mathbb{R}^{n+1} \rightarrow \mathbb{R}^{m}$. As a consequence they deduced the inequalities $N\left(S^{n}\right) \leq 3 n+2$ and $N\left(S^{2 k+1}\right) \leq$ $3(2 k+1)+1$.

It appears that very little is known about the exact values of $N(M)$. Indeed, according to $\underline{3}$, the only currently known exact values of this invariant are

$$
N\left(\mathbb{R}^{1}\right)=3, \quad N\left(S^{1}\right)=4, \quad N\left(\mathbb{R}^{2}\right)=6 .
$$

Finally for a general $n$-manifold $M^{n}$, Ghomi and Tabachnikov established upper and lower bounds

$$
2 n+1 \leq N\left(M^{n}\right) \leq 4 n+1
$$

and showed that the lower bound can be improved to $2 n+2$ if $M^{n}$ is a closed manifold.

In this paper we are interested in topological obstructions to totally skew embeddings of manifolds, in particular we address the problem of finding good lower bounds for $N\left(M^{n}\right)$. We demonstrate that in many classes of manifolds there are examples where the upper bound $4 n+1$ from (1) is very close to the actual value of $N\left(M^{n}\right)$. For example $N\left(\mathbb{R} P^{n}\right)$ is, by Proposition 5 , one of the numbers $4 n-1,4 n, 4 n+1$ if $n=2^{k}$ is a power of 2 , in particular $N\left(\mathbb{R} P^{2}\right)$ is 7,8 , or 9 . More generally, if $M^{n}=\mathbb{R} P^{n_{1}} \times \cdots \times \mathbb{R} P^{n_{k}}$ is a product of real projective spaces and $n_{i}=2^{r_{i}}$ are different powers of 2, then (Theorem 7)

$$
N\left(M^{n}\right)=N\left(\mathbb{R} P^{n_{1}} \times \cdots \times \mathbb{R} P^{n_{k}}\right) \geq 4 n-2 \alpha(n)+1,
$$

where $\alpha(n)$ is number of non-zero digits in the binary representation of $n$. A similar bound (Theorem 9)

$$
N(X) \geq 8 n-4 \alpha(n)+1=4 \cdot \operatorname{dim}_{\mathbb{R}}(X)-4 \alpha(n)+1
$$

is obtained if $X=\mathbb{C} P^{n_{1}} \times \cdots \times \mathbb{C} P^{n_{k}}$, where $n_{i}=2^{r_{i}}$ are different powers of 2 and $n=n_{1}+\cdots+n_{k}=\operatorname{dim}_{\mathbb{C}}(X)$.

In pursuit of other examples of manifolds where $N\left(M^{n}\right)$ gets very close to the upper bound $4 n+1$, we continue with the analysis of Grassmann manifolds $G_{k}\left(\mathbb{R}^{n+k}\right)$ and their oriented counterparts $\tilde{G}_{k}\left(\mathbb{R}^{n+k}\right)$. For example (Theorems 11 and 14) we prove that $N\left(G_{2}\left(\mathbb{R}^{2^{r}+2}\right)\right) \geq 4 \cdot 2^{r+1}-3$ and $N\left(\tilde{G}_{2}\left(\mathbb{R}^{2^{r}+2}\right)\right) \geq 3 \cdot 2^{r+1}+1$. Similar inequalities can be expected for many other Grassmannians as illustrated by the inequalities

$$
N\left(G_{3}\left(\mathbb{R}^{6}\right)\right) \geq 31, \quad N\left(G_{3}\left(\mathbb{R}^{7}\right)\right) \geq 43, \quad N\left(\tilde{G}_{3}\left(\mathbb{R}^{7}\right)\right) \geq 41, \text { etc. }
$$

These results are in sharp contrast with the fact that very little is known about the exact values of the invariant $N\left(M^{n}\right)$; for example, the exact value of $N\left(M^{2}\right)$ is not known for any closed surface $M^{2}$. This and a sample of other open problems and conjectures can be found in the final section of the paper where we also offer a brief outlook to future research.

Possibly the most intriguing and attractive is Conjecture 20 which, in analogy with the classical Immersion Conjecture 2, predicts that for $n>1$

$$
N\left(M^{n}\right) \leq 4 n-2 \alpha(n)+1 .
$$




\section{VECTOR BUNDLE DECOMPOSITION}

Let $F_{2}(M):=M^{2} \backslash \Delta_{M}$ be the configuration space (manifold) of all distinct ordered pairs of points in $M$. The tangent bundle $T\left(F_{2}(M)\right)$ admits a splitting

$$
T\left(F_{2}(M)\right) \cong \pi_{1}^{*} T M \oplus \pi_{2}^{*} T M,
$$

where $\pi_{1}, \pi_{2}: F_{2}(M) \rightarrow M$ are the natural projections. Simplifying the notation, let $T_{(x, y)}\left(F_{2}(M)\right) \cong T_{x}(M) \oplus T_{y}(M)$ be the fibre of this bundle at $(x, y) \in F_{2}(M)$.

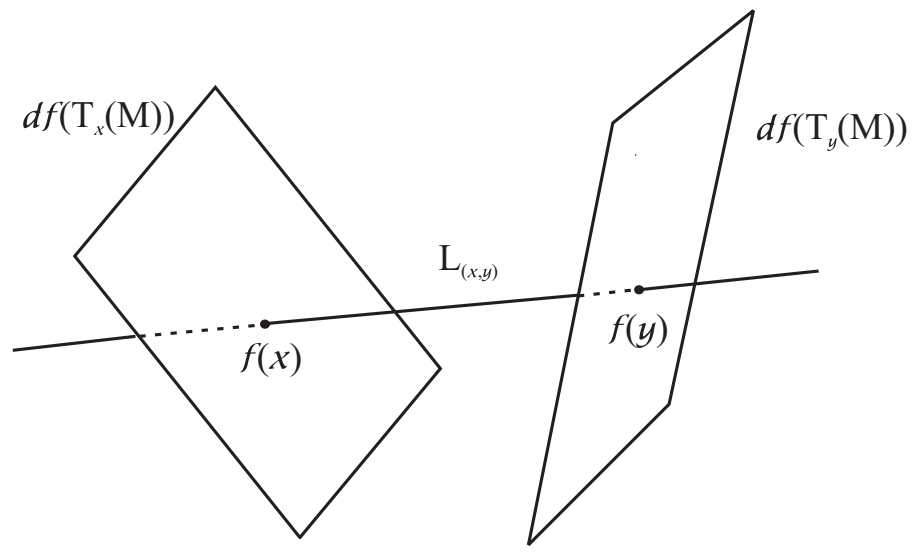

Figure 1. Fibre of the bundle $T\left(F_{2}(M)\right) \oplus \varepsilon^{1}$.

If $f: M^{n} \rightarrow \mathbb{R}^{N}$ is an embedding, then there is a trivial line bundle $L$ over $F_{2}(M)$ such that for $(x, y) \in F_{2}(M)$ the fibre $L_{(x, y)}$ is the line $\mathbb{R} \cdot(f(y)-f(x))$. If $f: M^{n} \rightarrow \mathbb{R}^{N}$ is a totally skew embedding, then there arises a monomorphism of vector bundles

$$
\Phi=\Phi^{(1)} \oplus \Phi^{(2)}: T\left(F_{2}(M)\right) \oplus \varepsilon^{1} \longrightarrow F_{2}(M) \times \mathbb{R}^{N},
$$

where $\Phi_{(x, y)}^{(1)}: T_{x}(M) \oplus T_{y}(M) \rightarrow \mathbb{R}^{N}$ is the map defined by $\Phi_{(x, y)}^{(1)}(u, v)=d f_{x}(u)+$ $d f_{y}(v)$ and $\Phi^{(2)}$, defined by $\Phi^{(2)}(\lambda)=\lambda(f(y)-f(x))$, maps the trivial line bundle $\varepsilon^{1}$ to $L$. In this case the trivial $N$-dimensional bundle $\varepsilon^{N}$ over $F_{2}(M)$ splits as

$$
\varepsilon^{N} \cong T\left(F_{2}(M)\right) \oplus \varepsilon^{1} \oplus \nu,
$$

where $\nu$ is a $(N-2 n-1)$-dimensional "normal" bundle. As a consequence ([7, Section 4]) we obtain the following proposition.

Proposition 1. If the dual Stiefel-Whitney class

$$
\bar{w}_{k}\left(T\left(F_{2}(M)\right)\right):=w_{k}(\nu) \in H^{k}\left(F_{2}(M) ; \mathbb{F}_{2}\right)
$$

is non-zero, then $2 n+k+1 \leq N$. In particular, $N(M) \geq 2 n+k+1$.

\section{Characteristic classes of $T\left(F_{2}(M)\right)$}

The cohomology of $F_{2}(M)=M^{2} \backslash \Delta_{M}$ can be calculated from the long exact sequence of the pair $\left(M^{2}, M^{2} \backslash \Delta_{M}\right)$,

$$
\cdots \longrightarrow H^{*}\left(M^{2}, M^{2} \backslash \Delta_{M}\right) \stackrel{\alpha}{\longrightarrow} H^{*}\left(M^{2}\right) \stackrel{\beta}{\longrightarrow} H^{*}\left(F_{2}(M)\right) \longrightarrow \cdots .
$$


We are interested in the (dual) Stiefel-Whitney classes (Proposition 1) so we tacitly assume that all cohomology has coefficients $\mathbb{F}_{2}$ unless otherwise noted.

By naturality, in order to check non-triviality of $\bar{w}_{k}\left(T\left(F_{2}(M)\right)\right)$, it is sufficient to check that $\bar{w}_{k}\left(M^{2}\right)$ is not in the image of the map $\alpha$.

The image $A:=\operatorname{Image}(\alpha)$ of $\alpha$ is determined in [7, Theorem 11.11] (see also [1, Chapter VI, Section 12]). It is generated, as an $H^{*}(M)$-module, by the "diagonal cohomology class"

$$
u^{\prime \prime}=\sum_{i=1}^{r} b_{i} \times b_{i}^{\sharp},
$$

where $\left\{b_{i}\right\}_{i=1}^{r}$ is an additive basis of $H^{*}(M)$ and $b_{i}^{\sharp}$ the class dual to $b_{i}$.

There are two actions of the ring $H^{*}(M)$ on $H^{*}(M \times M)$ associated with the projections $\pi_{1}, \pi_{2}: M^{2} \rightarrow M$. However in light of [7, Lemma 11.8], which says that if $a \in H^{*}(M)$, then

$$
(a \times 1) \cup u^{\prime \prime}=(1 \times a) \cup u^{\prime \prime},
$$

these two actions have the same effect on $u^{\prime \prime}$. As a consequence we obtain the following proposition.

\section{Proposition 2.}

$$
\begin{aligned}
A=\operatorname{Image}(\alpha)=H^{*}(M) \cdot u^{\prime \prime} & =\left\{(1 \times a) \cup u^{\prime \prime} \mid a \in H^{*}(M)\right\} \\
& =\left\{(a \times 1) \cup u^{\prime \prime} \mid a \in H^{*}(M)\right\} .
\end{aligned}
$$

The following proposition provides a simple and efficient criterion for testing if a class is in the image of the map $\alpha$. Note that the condition $k \leq n-1$ is essential since

$$
H^{2 n}(M \times M) \cong H^{n}(M) \otimes H^{n}(M) \subset \operatorname{Image}(\alpha) .
$$

Proposition 3. Let $M$ be a closed and smooth $n$-dimensional manifold. Let $k \leq$ $n-1$, and assume that $\theta \in H^{k}(M) \otimes H^{k}(M) \subset H^{*}(M \times M) \cong H^{*}(M) \otimes H^{*}(M)$ is a non-zero class. Then $\theta \notin \operatorname{Image}(\alpha)$. More generally, if $\omega \in H^{n+p}(M \times M)$ is a non-zero class which is in the image of $\alpha$, then it must have as a component of bidegree $(p, n)$ a non-zero "edge class" of the form $a \times z$ for some $a \in H^{p}(M)$, where $z \in H^{n}(M)$ is the fundamental cohomology class of $M$.

Proof. If $z \in H^{n}(M)$ is the fundamental cohomology class of $M$, then the diagonal class $u^{\prime \prime}$ has the form

$$
u^{\prime \prime}=z \times 1+x_{1} \times y_{1}+\cdots+x_{r} \times y_{r}+1 \times z,
$$

where $x_{i} \times y_{i}$ is a class of bidegree $(t, n-t)$ for some $0<t<n$. If $\omega \in H^{n+p}(M \times M)$ is in the image of $\alpha$, then we deduce from Proposition 2 that

$$
\omega=(a \times 1) u^{\prime \prime}=a z \times 1+A+a \times z,
$$

where $A=a x_{1} \times y_{1}+\cdots$ is a class whose homogeneous components are of bidegree $(q, n+p-q)$ for some $q>p$, and the proposition follows.

Corollary 4. If $k:=\max \left\{i \mid \bar{w}_{i}(M) \neq 0\right\}$, then $\bar{w}_{2 k}\left(T\left(F_{2}(M)\right)\right)=w_{2 k}(\nu) \neq 0$.

Proof. It follows from the naturality of Stiefel-Whitney classes that

$$
w_{2 k}(\nu)=\bar{w}_{2 k}\left(T\left(F_{2}(M)\right)\right)=\beta\left(\bar{w}_{2 k}\left(M^{2}\right)\right)=\beta\left(\bar{w}_{k}(M) \times \bar{w}_{k}(M)\right) .
$$

We observe that $k \leq n-1$. Indeed, each $n$-dimensional smooth manifold can be embedded in $\mathbb{R}^{2 n}$ and $\bar{w}_{n}(M)=0$ by [7, Corollary 11.4.]. 
Since $k \leq n-1$, we are allowed to use Proposition 3 which implies that $\bar{w}_{k}(M) \times$ $\bar{w}_{k}(M) \notin$ Image $(\alpha)$. From here and the exactness of the sequence (4) we finally deduce that $w_{2 k}(\nu) \neq 0$.

\section{ReAL PROJECTIVE SPACES}

As a first application, let us analyze the case when $M=\mathbb{R} P^{n}$ is the $n$-dimensional real projective space.

The cohomology algebra

$$
H^{*}\left(\mathbb{R} P^{n}\right) \cong \mathbb{F}_{2}[t] /\left(t^{n+1}=0\right)
$$

is a truncated polynomial ring with one generator $t \in H^{1}\left(\mathbb{R} P^{n}\right)$.

The total Stiefel-Whitney class of $T\left(\mathbb{R} P^{n}\right)$ is given ([7, Theorem 4.5.]) by the formula

$$
w\left(\mathbb{R} P^{n}\right)=(1+t)^{n+1}
$$

and the dual classes are

$$
\bar{w}\left(\mathbb{R} P^{n}\right)=w\left(\mathbb{R} P^{n}\right)^{-1} .
$$

Suppose that $n=2^{r}$ is a power of 2 . Then

$$
w\left(\mathbb{R} P^{n}\right)=1+t+t^{n} \quad \text { and } \quad \bar{w}\left(\mathbb{R} P^{n}\right)=1+t+t^{2}+\cdots+t^{n-1} .
$$

It follows that

$$
\bar{w}_{n-1}\left(\mathbb{R} P^{n}\right)=t^{n-1} \neq 0 .
$$

As a consequence of Corollary 4 , we obtain that $\bar{w}_{2 n-2}\left(F_{2}\left(\mathbb{R} P^{n}\right)\right) \neq 0$ and deduce from Proposition 1 the following result.

Proposition 5. If $f: \mathbb{R} P^{n} \rightarrow \mathbb{R}^{N}$ is a totally skew embedding and $n=2^{r}$ for some $r$, then

$$
N \geq 4 n-1
$$

Corollary 6. For each integer $n$,

$$
N\left(\mathbb{R} P^{n}\right) \geq 4 \cdot 2^{\left[\log _{2} n\right]}-1 .
$$

It follows from Proposition 5 and the inequalities (1) that if $n=2^{r}$ is a power of 2 , then $N\left(\mathbb{R} P^{n}\right)$ is $4 n-1,4 n$ or $4 n+1$, in particular $N\left(\mathbb{R} P^{2}\right)$ is 7,8 , or 9 .

\section{Products of Real projective spaces}

Suppose that $X=\mathbb{R} P^{n_{1}} \times \cdots \times \mathbb{R} P^{n_{k}}$ is a product of real projective spaces, where each $n_{i}=2^{r_{i}}$ is a power of 2 . Let $n=\operatorname{dim}(X)=n_{1}+\cdots+n_{k}$.

The cohomology $H^{*}(X) \cong \mathbb{F}_{2}\left[u_{1}, \ldots, u_{k}\right] /\left(u_{1}^{n_{1}+1}=\cdots=u_{k}^{n_{k}+1}=0\right)$ of $X$ is a truncated polynomial algebra with $k$ generators $u_{1}, \ldots, u_{k} \in H^{1}(X)$. Since $T(X)=T\left(\mathbb{R} P^{n_{1}}\right) \times \cdots \times T\left(\mathbb{R} P^{n_{k}}\right)$, the total Stiefel-Whitney class of $T(X)$ is given by the formula

$$
w(X)=\left(1+u_{1}\right)^{n_{1}+1} \cdots\left(1+u_{k}\right)^{n_{k}+1},
$$

and its dual total class is $\bar{w}(X)=w(X)^{-1}$.

By assumption, all integers $n_{i}$ are powers of 2 , hence

$$
w(X)=\left(1+u_{1}+u_{1}^{n_{1}}\right) \cdots\left(1+u_{k}+u_{k}^{n_{k}}\right)
$$

and the dual class has the form

$$
\bar{w}(X)=\left(1+u_{1}+\cdots+u_{1}^{n_{1}-1}\right) \cdots\left(1+u_{k}+\cdots+u_{k}^{n_{k}-1}\right) .
$$


From here we deduce that $\bar{w}_{n-k}=u_{1}^{n_{1}-1} \cdots u_{k}^{n_{k}-1}$ is non-zero and observe, by a reference to Proposition 3 and Corollary 4 that $\bar{w}_{2 n-2 k}\left(F_{2}(X)\right) \neq 0$. This fact allows us to use Proposition [1, which in turn implies the following theorem.

Theorem 7. Suppose that $X=\mathbb{R} P^{n_{1}} \times \cdots \times \mathbb{R} P^{n_{k}}$, where $n_{i}=2^{r_{i}}$ are powers of 2. Let $n=\operatorname{dim}(X)=n_{1}+\cdots+n_{k}$. If there exists a totally skew embedding of $X$ in $\mathbb{R}^{N}$, then $N \geq 4 n-2 k+1$. In particular if $n_{i} \neq n_{j}$ for $i \neq j$,

$$
N(X) \geq 4 n-2 \alpha(n)+1 \text {, }
$$

where $\alpha(n)$ is the number of non-zero digits in the binary representation of $n$.

\section{Complex MANifolds}

In some cases, for example if $M$ is a complex manifold, it may be convenient to use Pontryagin classes for estimating the invariant $N(M)$. However, the inequalities obtained by the use of Pontryagin classes are in general not as sharp as the inequalities obtained with the aid of Stiefel-Whitney classes, so we focus on the latter method.

6.1. Complex projective spaces. The cohomology of the complex projective space with $\mathbb{Z}$ coefficients is a truncated polynomial algebra, so by the Universal Coefficient Theorem we have $H^{*}\left(\mathbb{C} P^{n} ; \mathbb{F}_{2}\right) \cong \mathbb{F}_{2}[t] /\left(t^{n+1}=0\right)$, where $\operatorname{deg}(t)=2$.

Since the second Stiefel-Whitney class $w_{2}$ of any oriented 2-plane bundle is the $\bmod 2$ reduction of the Euler class, we observe that $t=w_{2}\left(\xi_{\mathbb{R}}\right)=w_{2}\left(\xi_{\mathbb{R}}^{*}\right)$, where $\xi$ is the canonical complex line bundle over $\mathbb{C} P^{n}$ and $\xi_{\mathbb{R}}$ is the underlying real 2-plane bundle.

The complex tangent bundle of the projective space $\mathbb{C} P^{n}$ is

$$
T\left(\mathbb{C} P^{n}\right) \cong \operatorname{Hom}\left(\xi, \xi^{\perp}\right),
$$

where $\xi^{\perp}$ is the complex $n$-plane bundle, complementary to the tautological complex line bundle $\xi$. Since $\operatorname{Hom}(\xi, \xi) \cong \varepsilon_{\mathbb{C}}^{1}$ is a trivial complex line bundle, we conclude that

$$
T\left(\mathbb{C} P^{n}\right) \oplus \varepsilon_{\mathbb{C}}^{1} \cong\left(\xi^{*}\right)^{\oplus(n+1)},
$$

where $\xi^{*}$ is the line bundle dual to $\xi$. By forgetting the complex structure (realification), we obtain the isomorphism of real bundles

$$
T\left(\mathbb{C} P^{n}\right)_{\mathbb{R}} \oplus \varepsilon_{\mathbb{R}}^{2} \cong\left(\xi_{\mathbb{R}}^{*}\right)^{\oplus(n+1)} .
$$

It follows that the total Stiefel-Whitney class of $T\left(\mathbb{C} P^{n}\right)_{\mathbb{R}}$ is

$$
w=w\left(T\left(\mathbb{C} P^{n}\right)_{\mathbb{R}}\right)=(1+t)^{n+1}=\left(1+w_{2}\right)^{n+1},
$$

where $w_{2}=w_{2}\left(\xi_{\mathbb{R}}\right)=w_{2}\left(\xi_{\mathbb{R}}^{*}\right)=t$ is the second Stiefel-Whitney class of the realification of the canonical bundle $\xi$.

Consequently, the dual Stiefel-Whitney class is

$$
\bar{w}=\left(1+w_{2}\right)^{-n-1}=\sum_{j=0}^{n}\left(\begin{array}{c}
n+j \\
j
\end{array}\right) w_{2}^{j} .
$$

We observe that the top class $\bar{w}_{2 n}$ is always zero, which is an instance of a much more general result of Massey (Theorem [19). Following Corollary 4, we search for the largest value of $j$ such that $\bar{w}_{2 j}=\left(\begin{array}{c}n+j \\ j\end{array}\right) w_{2}^{j} \neq 0$. We observe that $\bar{w}_{2 n-2} \neq 0$ precisely when $n=2^{r}$ is a power of 2 . 
Again, by invoking Corollary 4 , we conclude that $\bar{w}_{4 n-4}(\nu) \neq 0$, and finally by Proposition 1 we obtain the following result.

Theorem 8. Suppose that $n=2^{r}$ for some $r \geq 0$. Then

$$
N\left(\mathbb{C} P^{n}\right) \geq 4 n+(4 n-4)+1=4 \cdot \operatorname{dim}\left(\mathbb{C} P^{n}\right)-3 .
$$

6.2. Products of complex projective spaces. Suppose that $X=\mathbb{C} P^{n_{1}} \times \cdots \times$ $\mathbb{C} P^{n_{k}}$. As in Section 5 we focus on the case when $n_{i}=2^{r_{i}}$ for some $r_{i}$. As before $n=(1 / 2) \operatorname{dim}(X)=n_{1}+\cdots+n_{k}$. The cohomology ring of the space $X$ with $\mathbb{F}_{2}$ coefficients is

$$
H^{*}(X) \cong \mathbb{F}_{2}\left[u_{1} \ldots, u_{k}\right] /\left(u_{1}^{n_{1}+1}=\cdots=u_{k}^{n_{k}+1}=0\right),
$$

where $\operatorname{deg}\left(u_{1}\right)=\cdots=\operatorname{deg}\left(u_{n_{k}}\right)=2$.

We have already observed in Section 6.1 that if $n=2^{r}$, then

$$
\bar{w}\left(T\left(\mathbb{C} P^{n}\right)\right)=\sum_{j=0}^{n}\left(\begin{array}{c}
n+j \\
j
\end{array}\right) t^{j}=1+t+\cdots+t^{n-1} .
$$

It follows, as in Section [5, that the total dual Stiefel-Whitney class of $T(X)$ has the form

$$
\bar{w}(X)=\left(1+u_{1}+\cdots+u_{1}^{n_{1}-1}\right) \cdots\left(1+u_{k}+\cdots+u_{k}^{n_{k}-1}\right) .
$$

We conclude that $\bar{w}_{2 n-2 k}=u_{1}^{n_{1}-1} \cdots u_{k}^{n_{k}-1}$ is non-trivial, and as a consequence of Proposition 1 and Corollary 4, we obtain the following estimate.

Theorem 9. Suppose that $X=\mathbb{C} P^{n_{1}} \times \cdots \times \mathbb{C} P^{n_{k}}$, where $n_{i}=2^{r_{i}}$ are powers of 2 , and let $n=\operatorname{dim}_{\mathbb{C}}(X)=(1 / 2) \operatorname{dim}_{\mathbb{R}}(X)=n_{1}+\cdots+n_{k}$. Then $N(X) \geq 8 n-4 k+1$. In particular if all integers $n_{i}$ are distinct,

$$
N(X) \geq 8 n-4 \alpha(n)+1=4 \cdot \operatorname{dim}_{\mathbb{R}}(X)-4 \alpha(n)+1,
$$

where $\alpha(n)$ is the number of non-zero digits in the binary representation of $n$.

\section{Grassmannians}

We illustrate our method also for some cases of the Grassmann manifold $G_{k}\left(\mathbb{R}^{n+k}\right)$ of $k$-dimensional subspaces of $\mathbb{R}^{n+k}$ and some cases of the oriented Grassmann manifold $\tilde{G}_{k}\left(\mathbb{R}^{n+k}\right)$ of oriented $k$-dimensional subspaces of $\mathbb{R}^{n+k}$.

Let $\gamma_{k}$ be the canonical vector bundle over $X=G_{k}\left(\mathbb{R}^{n+k}\right)$ and $\tau$ the tangent bundle. Then from the relation $\tau \oplus \operatorname{Hom}\left(\gamma_{k}, \gamma_{k}\right) \cong(n+k) \gamma_{k}$, we obtain

$$
w(X) \cdot w\left(\gamma_{k} \otimes \gamma_{k}^{*}\right)=w\left(\gamma_{k}\right)^{n+k},
$$

where $w\left(\gamma_{k} \otimes \gamma_{k}^{*}\right)=p_{k}\left(w_{1}, \ldots, w_{k}\right), p_{k}$ is the polynomial over $\mathbb{F}_{2}$ defined by

$$
p_{k}\left(\sigma_{1}, \ldots, \sigma_{k}\right)=\prod_{i=1}^{k} \prod_{j=1}^{k}\left(1+x_{i}+x_{j}\right),
$$

and $\sigma_{1}, \ldots, \sigma_{k}$ are the elementary symmetric polynomials in variables $x_{1}, \ldots, x_{k}$; see [7, Problem 7C].

In the special case when $k=2$ and $k=3$, by a direct computation we check that $w\left(\gamma_{2} \otimes \gamma_{2}^{*}\right)=1+w_{1}^{2}$ and $w\left(\gamma_{3} \otimes \gamma_{3}^{*}\right)=1+w_{1}^{4}+w_{2}^{2}+w_{1}^{2} w_{2}^{2}+w_{3}^{2}$.

Since $w\left(\gamma_{k}\right)=1+w_{1}+w_{2}+\cdots+w_{k}$, it follows that the total Stiefel-Whitney class of the complementary bundle to the tangent bundle $\tau$ equals

$$
\bar{w}(X)=w\left(\gamma_{k} \otimes \gamma_{k}^{*}\right)\left(1+w_{1}+w_{2}+\cdots+w_{k}\right)^{-(n+k)} .
$$


Completely analogous formulae are true in the case of the oriented Grassmann manifold $\tilde{X}=\tilde{G}_{k}\left(\mathbb{R}^{n+k}\right)$, the only difference being the vanishing of the first StiefelWhitney class, $w_{1}\left(\tilde{\gamma}_{k}\right)=0$.

7.1. $G_{k}\left(\mathbb{R}^{n+k}\right)$. First we treat the case $k=2$ and $n=2^{r}$, that is the case of the Grassmann manifold $G_{2}\left(\mathbb{R}^{2^{r}+2}\right)$. We shall need the following lemma.

Lemma 10. The class $w_{1}^{2} w_{2}^{2^{r}-2} \in H^{2^{r+1}-2}\left(G_{2}\left(\mathbb{R}^{2^{r}+2}\right)\right)$ is non-trivial.

Proof. Let us assume, to the contrary, that $w_{1}^{2} w_{2}^{2^{r}-2}=0$. Then $w_{1}^{2} w_{2}^{2^{r}-1}=0$. Since the map

$$
H^{2^{r+1}-1}\left(G_{2}\left(\mathbb{R}^{2^{r}+2}\right)\right) \stackrel{\cup w_{7}}{\longrightarrow} H^{2^{r+1}}\left(G_{2}\left(\mathbb{R}^{2^{r}+2}\right)\right)
$$

is an isomorphism by Poincaré duality, it follows that $w_{1} w_{2}^{2^{r}-1}=0$.

We show that $w_{1}^{2^{r+1}-2}$ and $w_{2}^{2^{r}-1}$ are non-trivial classes in $H^{2^{r+1}-2}\left(G_{2}\left(\mathbb{R}^{2^{r}+2}\right)\right)$. The first observation is a consequence of a result of Stong [11] about the height of $w_{1}$, which in this case is $\operatorname{ht}\left(w_{1}\right)=2^{r+1}-2$. The second observation follows from the well-known fact that $w_{k}^{n}$ is a non-trivial element in $H^{k n}\left(G_{k}\left(\mathbb{R}^{k+n}\right)\right)$. Let us show that these two classes are different. We have

$$
S q^{2}\left(w_{1}^{2^{r+1}-2}\right)=\left(\begin{array}{c}
2^{r+1}-2 \\
2
\end{array}\right) w_{1}^{2^{r+1}}=0,
$$

again by the same result of Stong. By the $\mathrm{Wu}$ formula $S q^{1}\left(w_{2}\right)=w_{1} w_{2}$ (see [7, Problem 8A]), we have

$$
S q^{2}\left(w_{2}^{2^{r}-1}\right)=\left(2^{r}-1\right) w_{2}^{2^{r}}+\left(\begin{array}{c}
2^{r}-1 \\
2
\end{array}\right) w_{1}^{2} w_{2}^{2^{r}-1}=w_{2}^{2^{r}} \neq 0 .
$$

So, $H^{2^{r+1}-2}\left(G_{2}\left(\mathbb{R}^{2^{r}+2}\right)\right) \cong \mathbb{Z} / 2 \oplus \mathbb{Z} / 2$ is generated by $w_{1}^{2^{r+1}-2}$ and $w_{2}^{2^{r}-1}$.

The map $\phi: H^{2^{r+1}-2}\left(G_{2}\left(\mathbb{R}^{2^{r}+2}\right)\right) \stackrel{\bigcup w_{1}}{\longrightarrow} H^{2^{r+1}-1}\left(G_{2}\left(\mathbb{R}^{2^{r}+2}\right)\right)$ satisfies the relations $\phi\left(w_{1}^{2^{r+1}-2}\right)=w_{1}^{2^{r+1}-1}=0$ and $\phi\left(w_{2}^{2^{r}-1}\right)=w_{1} w_{2}^{2^{r}-1}=0$, as we proved in the beginning of the proof. So, $\phi=0$. This is a contradiction, since $2^{r+1}-1$ is odd and $H^{2^{r+1}-1}\left(G_{2}\left(\mathbb{R}^{2^{r}+2}\right)\right) \cong \mathbb{Z} / 2$ could be generated only by the element of the type $w_{1}^{2 s-1} w_{2}^{t}$. So, our assumption is false, and we have $w_{1}^{2} w_{2}^{2^{r}-2} \neq 0$.

Theorem 11. $N\left(G_{2}\left(\mathbb{R}^{2^{r}+2}\right)\right) \geq 4 \cdot 2^{r+1}-3$.

Proof. The total Stiefel-Whitney class of the normal bundle of $X=G_{2}\left(\mathbb{R}^{2^{r}+2}\right)$ equals, by equation (15),

$$
\begin{aligned}
\bar{w}(X) & =\left(1+w_{1}^{2}\right)\left(1+w_{1}+w_{2}\right)^{-\left(2^{r}+2\right)} \\
& =\left(1+w_{1}^{2}\right)\left(1+w_{1}+w_{2}\right)^{-2^{r+1}}\left(1+w_{1}+w_{2}\right)^{2^{r}-2} \\
& =\left(1+w_{1}^{2}\right)\left(1+w_{1}^{2^{r+1}}\right)\left(1+w_{1}+w_{2}\right)^{2^{r}-2} \\
& =\left(1+w_{1}^{2}\right)\left(1+w_{1}+w_{2}\right)^{2^{r}-2} .
\end{aligned}
$$

It follows that $\bar{w}_{2^{r+1}-2}=w_{1}^{2} w_{2}^{2^{r}-2} \neq 0$, which by Corollary 4 and Proposition 1 implies the inequality

$$
N\left(G_{2}\left(\mathbb{R}^{2^{r}+2}\right)\right) \geq 4 \cdot 2^{r+1}-3=4 \cdot \operatorname{dim} G_{2}\left(\mathbb{R}^{2^{r}+2}\right)-3 .
$$


As an illustration of our methods in the case $k>2$, we outline the computations in the particular case of the Grassmann manifold $G_{3}\left(\mathbb{R}^{7}\right)$.

Theorem 12. $N\left(G_{3}\left(\mathbb{R}^{7}\right)\right) \geq 43$.

Proof. The cohomology of $X=G_{3}\left(\mathbb{R}^{7}\right)$ is generated by the Stiefel-Whitney classes $w_{1}, w_{2}, w_{3}$ subject to the relation $\left(1+w_{1}+w_{2}+w_{3}\right)\left(1+\bar{w}_{1}+\bar{w}_{2}+\bar{w}_{3}+\bar{w}_{4}\right)=1$. It follows that $\bar{w}_{1}=w_{1}, \bar{w}_{2}=w_{1}^{2}+w_{2}, \bar{w}_{3}=w_{1}^{3}+w_{3}$, and $\bar{w}_{4}=w_{1}^{4}+w_{1}^{2} w_{2}+w_{2}^{2}$. Moreover, $R_{1}:=w_{1}^{5}+w_{1} w_{2}^{2}+w_{1}^{2} w_{3}=0, R_{2}:=w_{1}^{4} w_{2}+w_{1}^{2} w_{2}^{2}+w_{2}^{3}+w_{1}^{3} w_{3}+w_{3}^{2}=0$, and $R_{3}:=w_{1}^{4} w_{3}+w_{1}^{2} w_{2} w_{3}+w_{2}^{2} w_{3}=0$.

It could be shown that a consequence of these relations is also the relation

$$
0=\left(w_{1}^{3}+w_{1} w_{2}+w_{3}\right) R_{1}+w_{1}^{2} R_{2}+w_{1} R_{3}=w_{1}^{8} .
$$

It requires a few more steps to show that the class $w_{1}^{2} w_{2}^{2} w_{3}+w_{3}^{3}=w_{1}^{5} w_{2}^{2}$ is non-trivial. It is actually one of the additive generators of the cohomology group $H^{9}\left(G_{3}\left(\mathbb{R}^{7}\right)\right)$.

The total Stiefel-Whitney class of the bundle complementary to the tangent bundle equals

$$
\begin{aligned}
\bar{w}(X) & =\left(1+w_{1}+w_{2}+w_{3}\right)^{-7} \cdot\left(1+w_{1}^{4}+w_{2}^{2}+w_{1}^{2} w_{2}^{2}+w_{3}^{2}\right) \\
& =\left(1+w_{1}+w_{2}+w_{3}\right)\left(1+w_{1}+w_{2}+w_{3}\right)^{-8}\left(1+w_{1}^{4}+w_{2}^{2}+w_{1}^{2} w_{2}^{2}+w_{3}^{2}\right) \\
& =\left(1+w_{1}+w_{2}+w_{3}\right)\left(1+w_{1}^{4}+w_{2}^{2}+w_{1}^{2} w_{2}^{2}+w_{3}^{2}\right) .
\end{aligned}
$$

We already noticed that $\bar{w}_{9}(X)=w_{1}^{2} w_{2}^{2} w_{3}+w_{3}^{3}$ is a non-trivial class, and it is the top-dimensional one.

Altogether, we conclude that $N\left(G_{3}\left(\mathbb{R}^{7}\right)\right) \geq 24+1+18=43$.

Let us add that, in a similar way but more easily, one obtains by the same method also $N\left(G_{2}\left(\mathbb{R}^{5}\right)\right) \geq 21, N\left(G_{2}\left(\mathbb{R}^{7}\right)\right) \geq 29, N\left(G_{3}\left(\mathbb{R}^{6}\right)\right) \geq 31$, and $N\left(G_{3}\left(\mathbb{R}^{8}\right)\right) \geq 43$.

7.2. $\tilde{G}_{k}\left(\mathbb{R}^{n+k}\right)$. For comparison we include an analysis of some cases where the manifold $M$ is the Grassmannian of all oriented $k$-dimensional subspaces in $\mathbb{R}^{n+k}$.

Let us denote by $p: \tilde{G}_{k}\left(\mathbb{R}^{n+k}\right) \rightarrow G_{k}\left(\mathbb{R}^{n+k}\right)$ the two-fold covering. Then, $\tilde{w}_{i}=w_{i}\left(\tilde{G}_{k}\left(\mathbb{R}^{n+k}\right)\right)=p^{*}\left(w_{i}\left(G_{k}\left(\mathbb{R}^{n+k}\right)\right)\right)$, and we know that $\tilde{w}_{1}=0$. Since $\tilde{w}_{i}=$ $p^{*}\left(w_{i}\right) \neq 0$ implies $w_{i} \neq 0$, the estimates obtained in this way for the oriented Grassmann manifold $\tilde{G}_{k}\left(\mathbb{R}^{n+k}\right)$ cannot be better than those for $G_{k}\left(\mathbb{R}^{n+k}\right)$.

The cohomology ring of the oriented Grassmann manifold $H^{*}\left(\tilde{G}_{k}\left(\mathbb{R}^{n+k}\right)\right)$ is more complicated however, and it is more difficult to determine which Stiefel-Whitney classes are non-trivial in this case. Aside from triviality of $\tilde{w}_{1}$, we know that $H^{*}\left(\tilde{G}_{k}\left(\mathbb{R}^{n+k}\right)\right)$ has some additional generators and some additional relations.

Let $B_{n}^{k}=\left(w_{1}\right)$ be the principal ideal in $H^{*}\left(G_{2}\left(\mathbb{R}^{n+k}\right)\right)$ generated by $w_{1}$. In order to determine which Stiefel-Whitney classes are non-trivial in the oriented case, we use the calculations in $H^{*}\left(G_{k}\left(\mathbb{R}^{n+k}\right)\right)$ and the Gysin exact sequence in cohomology (cf. [7, Theorem 12.4]),

$$
\cdots \rightarrow H^{i-1}\left(G_{k}\left(\mathbb{R}^{n+k}\right)\right) \stackrel{\bigcup w_{1}}{\longrightarrow} H^{i}\left(G_{k}\left(\mathbb{R}^{n+k}\right)\right) \stackrel{p^{*}}{\longrightarrow} H^{i}\left(\tilde{G}_{k}\left(\mathbb{R}^{n+k}\right)\right) \rightarrow \cdots .
$$

From the exactness of this sequence, we deduce that for a given Stiefel-Whitney class $w_{i_{1}}^{j_{1}} \cdots w_{i_{r}}^{j_{r}} \in H^{*}\left(G_{k}\left(\mathbb{R}^{n+k}\right)\right), p^{*}\left(w_{i_{1}}^{j_{1}} \cdots w_{i_{r}}^{j_{r}}\right)=0$ if and only if $w_{i_{1}}^{j_{1}} \cdots w_{i_{r}}^{j_{r}} \in$ $B_{n}^{k}=\left(w_{1}\right)$.

Also, we easily check that in this case the polynomials $p_{2}$ and $p_{3}$ from the equation (14) reduce to $\tilde{p}_{2}\left(\tilde{w}_{2}\right)=1$ and $\tilde{p}_{3}\left(\tilde{w}_{2}, \tilde{w}_{3}\right)=1+\tilde{w}_{2}^{2}+\tilde{w}_{3}^{2}$. 
We now turn to the case $k=2$. Let us determine the height of the class $\tilde{w}_{2}$ in $H^{*}\left(\tilde{G}_{2}\left(\mathbb{R}^{n+2}\right)\right), \operatorname{ht}\left(\tilde{w}_{2}\right)=\max \left\{m \in \mathbb{N} \mid \tilde{w}_{2}^{m} \neq 0\right\}$.

In $H^{*}\left(G_{2}\left(\mathbb{R}^{n+2}\right)\right)$, we have $\left(1+w_{1}+w_{2}\right)\left(1+\bar{w}_{1}+\cdots+\bar{w}_{n}\right)=1$, and so

$$
\bar{w}_{r}=w_{1} \bar{w}_{r-1}+w_{2} \bar{w}_{r-2}, \quad 3 \leq r \leq n .
$$

If as before $B_{n}^{2}=\left(w_{1}\right)$ is the principal ideal in $H^{*}\left(G_{2}\left(\mathbb{R}^{n+2}\right)\right)$ generated by $w_{1}$, then inductively, using the relations (19), we show that

$$
\bar{w}_{2 k-1} \in B_{n}^{2}, \quad 2 k-1 \leq n
$$

and

$$
\bar{w}_{2 k} \equiv w_{2}^{k} \quad\left(\bmod B_{n}^{2}\right), \quad 2 k \leq n
$$

Note that in dimensions $\leq n$ there are no polynomial relations among $w_{1}$ and $w_{2}$.

Lemma 13. $\operatorname{ht}\left(\tilde{w}_{2}\right)=\left[\frac{n}{2}\right]$.

Proof. We already know that $\operatorname{ker} p^{*}=B_{n}^{2}$. The dimension of $w_{2}^{\left[\frac{n}{2}\right]}$ is $2 \cdot\left[\frac{n}{2}\right] \leq 2 \cdot \frac{n}{2}=$ $n$, hence this class cannot be written as a multiple of $w_{1}$ (for in dimensions $\leq n$ there are no relations among $w_{1}$ and $\left.w_{2}\right)$. Thus $w_{2}^{\left[\frac{n}{2}\right]} \notin \operatorname{ker} p^{*}$, and so $\tilde{w}_{2}^{\left[\frac{n}{2}\right]}=p^{*}\left(w_{2}^{\left[\frac{n}{2}\right]}\right) \neq 0$.

In order to show that $\tilde{w}_{2}^{\left[\frac{n}{2}\right]+1}=0$, we distinguish two cases.

If $n=2 l$, in dimension $2 l+2$ we have the relation $w_{2} \bar{w}_{2 l}=0$. But $\bar{w}_{2 l}=w_{2}^{l}+w_{1} \cdot u$ for some class $u$, so $w_{2}^{l+1}+w_{1} w_{2} u=0$ and $w_{2}^{l+1} \in B_{n}^{2}$. So we obtain

$$
\tilde{w}_{2}^{\left[\frac{n}{2}\right]+1}=\tilde{w}_{2}^{l+1}=p^{*}\left(w_{2}^{l+1}\right)=0 .
$$

If $n=2 l+1$, in dimension $2 l+2$ we have the relation $w_{1} \bar{w}_{2 l+1}+w_{2} \bar{w}_{2 l}=0$. The first summand belongs to $B_{n}^{2}$, so we show (as in the first case) that $w_{2}^{l+1} \in B_{n}^{2}$. Here we also have that $l=\left[\frac{n}{2}\right]$ and the lemma follows.

Let us also notice that by equation (15) and the fact that $\tilde{p}_{2}=1$, the total Stiefel-Whitney class of the complementary normal bundle to the tangent bundle of the space $X=\tilde{G}_{2}\left(\mathbb{R}^{n+2}\right)$ equals

$$
\bar{w}(X)=\left(1+\tilde{w}_{2}\right)^{-(n+2)}=\left(\left(1+\tilde{w}_{2}\right)^{-1}\right)^{n+2} .
$$

In the light of Lemma 13 we have

$$
\left(1+\tilde{w}_{2}\right)\left(1+\tilde{w}_{2}+\tilde{w}_{2}^{2}+\cdots+\tilde{w}_{2}^{\left[\frac{n}{2}\right]}\right)=1+\tilde{w}_{2}^{\left[\frac{n}{2}\right]+1}=1,
$$

and so $\left(1+\tilde{w}_{2}\right)^{-1}=1+\tilde{w}_{2}+\tilde{w}_{2}^{2}+\cdots+\tilde{w}_{2}^{\left[\frac{n}{2}\right]}$.

Finally, we obtain

$$
\bar{w}\left(\tilde{G}_{2}\left(\mathbb{R}^{n+2}\right)\right)=\left(1+\tilde{w}_{2}+\tilde{w}_{2}^{2}+\cdots+\tilde{w}_{2}^{\left[\frac{n}{2}\right]}\right)^{n+2} .
$$

Theorem 14. $N\left(\tilde{G}_{2}\left(\mathbb{R}^{2^{r}+2}\right)\right) \geq 3 \cdot 2^{r+1}+1=3 \cdot \operatorname{dim} \tilde{G}_{2}\left(\mathbb{R}^{2^{r}+2}\right)+1$.

Proof. Substituting $n=2^{r}$ in the above considerations and using Lemma 13, we have

$$
\begin{aligned}
\bar{w}\left(\tilde{G}_{2}\left(\mathbb{R}^{2^{r}+2}\right)\right) & =\left(1+\tilde{w}_{2}+\tilde{w}_{2}^{2}+\cdots+\tilde{w}_{2}^{2^{r-1}}\right)^{2^{r}+2} \\
& =\left(1+\tilde{w}_{2}+\tilde{w}_{2}^{2}+\cdots+\tilde{w}_{2}^{2^{r-1}}\right)^{2} \\
& =1+\tilde{w}_{2}^{2}+\tilde{w}_{2}^{4}+\cdots+\tilde{w}_{2}^{2^{r-1}} .
\end{aligned}
$$

By Lemma 13, $\tilde{w}_{2}^{2^{r-1}} \neq 0$, and by Corollary $4, N\left(\tilde{G}_{2}\left(\mathbb{R}^{2^{r}+2}\right)\right) \geq 1+2 \cdot 2^{r+1}+2$. $2^{r}=3 \cdot 2^{r+1}+1=3 \cdot \operatorname{dim} \tilde{G}_{2}\left(\mathbb{R}^{2^{r}+2}\right)+1$. 
Let us add that by the same methods one easily obtains $N\left(\tilde{G}_{2}\left(\mathbb{R}^{2^{r}+1}\right)\right) \geq 3$. $2^{r+1}-7=3 \cdot \operatorname{dim} \tilde{G}_{2}\left(\mathbb{R}^{2^{r}+1}\right)-1, N\left(\tilde{G}_{2}\left(\mathbb{R}^{2^{r}+3}\right)\right) \geq 3 \cdot 2^{r+1}+5=3 \cdot \operatorname{dim} \tilde{G}_{2}\left(\mathbb{R}^{2^{r}+3}\right)-1$, and $N\left(\tilde{G}_{2}\left(\mathbb{R}^{2^{r}+4}\right)\right) \geq 3 \cdot 2^{r+1}+9=3 \cdot \operatorname{dim} \tilde{G}_{2}\left(\mathbb{R}^{2^{r}+4}\right)-3$. It is also seen from the proof that our method cannot give better lower bounds in all these cases.

Let us now prove the result corresponding to Theorem 12 in the case of the oriented Grassmannian.

Theorem 15. $N\left(\tilde{G}_{3}\left(\mathbb{R}^{7}\right)\right) \geq 41$.

Proof. In the cohomology of the oriented Grassmannian $\tilde{X}=\tilde{G}_{3}\left(\mathbb{R}^{7}\right)$, we have

$$
\begin{aligned}
\bar{w}(\tilde{X})=p^{*}(\bar{w}(X)) & =\left(1+\tilde{w}_{2}+\tilde{w}_{3}\right)^{-7}\left(1+\tilde{w}_{2}^{2}+\tilde{w}_{3}^{2}\right) \\
& =\left(1+\tilde{w}_{2}+\tilde{w}_{3}\right)\left(1+\tilde{w}_{2}+\tilde{w}_{3}\right)^{-8}\left(1+\tilde{w}_{2}^{2}+\tilde{w}_{3}^{2}\right) \\
& =\left(1+\tilde{w}_{2}+\tilde{w}_{3}\right)\left(1+\tilde{w}_{2}^{2}+\tilde{w}_{3}^{2}\right) .
\end{aligned}
$$

In the previous subsection we showed that the class $\bar{w}_{9}(X)=w_{1}^{2} w_{2}^{2} w_{3}+w_{3}^{3}=$ $w_{1}^{5} w_{2}^{2}$ is non-trivial, but it is trivial in the cohomology of the oriented Grassmannian. However, it can be shown (using the computations in $H^{*}\left(G_{3}\left(\mathbb{R}^{7}\right)\right)$ ) that the class $\bar{w}_{8}(X)=w_{1}^{2} w_{2}^{3}+w_{2} w_{3}^{2}$ cannot be written as a product of $w_{1}$ with some other class. So,

$$
\bar{w}_{8}(\tilde{X})=\tilde{w}_{2} \tilde{w}_{3}^{2} \neq 0 .
$$

As a consequence, by Corollary $\llbracket$ we have $N\left(\tilde{G}_{3}\left(\mathbb{R}^{7}\right)\right) \geq 1+24+16=41$.

We end this section by presenting a slightly different method of calculation applied to the Grassmannian $Y=\tilde{G}_{3}\left(\mathbb{R}^{13}\right)$. In this case $\operatorname{dim}(Y)=30$ and

$$
\begin{aligned}
\bar{w}(Y) & =\left(1+\tilde{w}_{2}^{2}+\tilde{w}_{3}^{2}\right)\left(1+\tilde{w}_{2}+\tilde{w}_{3}\right)^{-13} \\
& =\left(1+\tilde{w}_{2}^{2}+\tilde{w}_{3}^{2}\right)\left(1+\tilde{w}_{2}+\tilde{w}_{3}\right)^{3}\left(1+\tilde{w}_{2}+\tilde{w}_{3}\right)^{-16} \\
& =\left(1+\tilde{w}_{2}^{2}+\tilde{w}_{3}^{2}\right)\left(1+\tilde{w}_{2}+\tilde{w}_{3}\right)^{3} \\
& =\tilde{w}_{3}^{5}+\tilde{w}_{2} \tilde{w}_{3}^{4}+\cdots,
\end{aligned}
$$

where dots represent some lower-dimensional classes. In order to check whether some of the classes $\bar{w}_{15}(Y)=\tilde{w}_{3}^{5}$ and $\bar{w}_{14}(Y)=\tilde{w}_{2} \tilde{w}_{3}^{4}$ are non-trivial, we use a criterion from [5]. It says that $w_{2}^{i_{2}} w_{3}^{i_{3}} \in H^{*}\left(G_{3}\left(\mathbb{R}^{13}\right)\right)$ cannot be expressed as a multiple of the class $w_{1}$ if it does not belong to the ideal $J_{n, 3}$ of $\mathbb{Z}_{2}\left[w_{2}, w_{3}\right]$ generated by the homogeneous components of

$$
\frac{1}{1+w_{2}+w_{3}}=\left(1+w_{2}+w_{3}\right)^{2^{r+3}-1}=\sum_{i=0}^{2^{r+3}-1} \sum_{j=0}^{i}\left(\begin{array}{l}
i \\
j
\end{array}\right) w_{2}^{i-j} w_{3}^{j}
$$

in dimensions $n-2, n-1, n$, which we respectively denote by $g_{n-2}, g_{n-1}, g_{n}$. The integer $r$ satisfies the inequalities $2^{r}<n \leq 2^{r+1}$, which for dimensional reasons leads to the desired relation $\left(1+w_{2}+w_{3}\right)^{2^{r+3}}=1$. Now it is not difficult to see that

$$
g_{k}=\sum_{k / 3 \leq i \leq k / 2}\left(\begin{array}{c}
i \\
3 i-k
\end{array}\right) w_{2}^{3 i-k} w_{3}^{k-2 i} .
$$

By an easy computation, $g_{13}=0, g_{12}=w_{3}^{4}+w_{2}^{6}$, and $g_{11}=w_{2}^{4} w_{3}$. It turns out that $w_{3}^{5}=w_{3} g_{12}+w_{2}^{2} g_{11} \in J_{n, 3}$, but $w_{2} w_{3}^{4} \notin J_{n, 3}$, since it does not belong to the span of $w_{2} g_{12}=w_{2} w_{3}^{4}+w_{2}^{7}$ and $w_{3} g_{11}=w_{2}^{4} w_{3}^{2}$. Consequently, $\bar{w}_{14}(Y)=\tilde{w}_{2} \tilde{w}_{3}^{4} \neq 0$, and by Corollary $4, N\left(\tilde{G}_{3}\left(\mathbb{R}^{13}\right) \geq 1+2 \cdot 30+2 \cdot 14=89\right.$. 


\section{CONCLUding REMARKS}

8.1. Embeddings with multiple regularity. The problem of estimating the invariant $N\left(M^{n}\right)$ was in 9 ] (see also [10]) incorporated in a more general question of studying $(k, l)$-regular embeddings. By definition a smooth map $f: M^{n} \rightarrow \mathbb{R}^{N}$ is $(k, l)$-regular if for every collection of $k+l$ distinct points $x_{1}, \ldots, x_{k}, y_{1}, \ldots, y_{l}$ in $M^{n}$ and $l$ tangent lines $L_{i} \subset T_{y_{i}}\left(M^{n}\right), i=1, \ldots, l$, the set of points and lines

$$
f\left(x_{1}\right), \ldots, f\left(x_{k}\right), d f\left(L_{1}\right), \ldots, d f\left(L_{l}\right)
$$

is affinely independent.

When $l=0$, the notion of $(k, l)$-regularity reduces to affine $(k-1)$-regularity in the sense of Handel and Segal [4]. On the other hand, a smooth map is $(0,2)$-regular if and only if it is totally skew.

The existence of a $(k, l)$-regular embedding $f: M^{n} \rightarrow \mathbb{R}^{N}$ implies, essentially by the arguments of Section 2, a splitting

$$
\varepsilon^{N} \cong \pi^{*} T\left(F_{l}(M)\right) \oplus \varepsilon^{k+l-1} \oplus \nu
$$

of the trivial $N$-dimensional bundle over the configuration space $F_{k+l}(M)$ of all ordered collections of $k+l$ distinct points in $M^{n}$. By definition $\pi^{*} T\left(F_{l}(M)\right)$ is the pullback of the tangent bundle $T\left(F_{l}(M)\right)$ along the projection map $\pi: F_{k+l}(M) \rightarrow$ $F_{l}(M)$ and $\nu$ is a bundle of dimension $N-(n+1) l-k+1$.

This is a clear indication that the problem of studying $(k, l)$-regular embeddings is amenable to the methods of Section 3 and we hope to return to this question in a subsequent publication.

8.2. Open problems. In this section we collect some open problems pointing to some of the most interesting questions about totally skew embeddings of manifolds.

Problem 16. Determine the exact values of $N\left(S^{2}\right)$ and $N\left(\mathbb{R} P^{2}\right)$. More generally what is the exact value of $N\left(M^{2}\right)$ if $M^{2}$ is a closed or open surface? According to [3], the only known result is $N(M)=6$, where $D^{2} \subset M \subset \mathbb{R}^{2}$.

An immersion $\phi: M^{n} \rightarrow \mathbb{R}^{N}$ is called totally skew if whenever $\phi(x) \neq \phi(y)$ the affine subspaces $d \phi\left(T_{x}\left(M^{n}\right)\right)$ and $d \phi\left(T_{y}\left(M^{n}\right)\right)$ are skew. If $f: M \rightarrow \mathbb{R}^{N}$ is a totally skew embedding and if $g: \widetilde{M} \rightarrow M$ is a covering map, then $\phi=f \circ g$ is clearly a totally skew immersion. The following conjecture reflects our impression that in this case a totally skew immersion can be perturbed to a genuine totally skew embedding.

Conjecture 17. If $M$ is a closed, smooth manifold and $\Gamma$ a finite group acting freely on $M$, then

$$
N(M) \leq N(M / \Gamma)
$$

in particular $N\left(S^{n}\right) \leq N\left(\mathbb{R} P^{n}\right)$.

It follows from the splitting (3) that the geometric dimension $g$-dim $(\nu)$ of the normal bundle $\nu_{1}=\nu\left(T\left(F_{2}(M)\right)\right)$ satisfies the inequality

$$
N\left(M^{n}\right)-1 \geq 2 n+g-\operatorname{dim}\left(\nu_{1}\right) .
$$

Similar inequalities hold for manifolds $X, Y$ and $X \times Y$ and their comparison suggests the possibility of the following general result. 
Conjecture 18. For two manifolds $X$ and $Y, N(X \times Y) \geq N(X)+N(Y)-1$.

This conjecture, if true, would, together with the bound $N\left(\mathbb{R}^{n}\right) \geq 3 n$ for $n$ a power of 2 (obtained in [3), imply the lower bound $N\left(\mathbb{R}^{n}\right) \geq 3 n-\alpha(n)+1$.

The well-known Immersion Conjecture, resolved positively by R. Cohen [2] in 1985 , predicted that any compact smooth $n$-manifold for $n>1$ can be immersed in $\mathbb{R}^{2 n-\alpha(n)}$, where $\alpha(n)$ is the number of non-zero digits in the binary representation of $n$. The following result of Massey, which preceded Cohen's theorem by 15 years, played an important role by providing strong evidence in favor of the conjecture.

Theorem 19 (W. S. Massey, 6]). Let $M^{n}$ be a smooth, compact n-dimensional manifold $(n>1)$. Then $\bar{w}_{j}(M)=0$ for $j>n-\alpha(n)$.

Theorem 19 together with our Corollary 4 provides interesting initial evidence for the following bold conjecture.

Conjecture 20. For every $n$-dimensional, compact smooth manifold $M^{n}(n>1)$,

$$
N\left(M^{n}\right) \leq 4 n-2 \alpha(n)+1 .
$$

If correct, Conjecture 20, together with Proposition [5 and Theorem 7 would yield some exact computations of the invariant $N\left(M^{n}\right)$. For example it would imply

$$
N\left(\mathbb{R} P^{2}\right)=7,
$$

and more generally the following result.

Conjecture 21. Suppose that $n_{i}=2^{r_{i}}(i=1, \ldots, k)$ and assume that $r_{i} \neq r_{j}$ for $i \neq j$. Let $n=n_{1}+\cdots+n_{k} \geq 2$. Then,

$$
N\left(\mathbb{R} P^{n_{1}} \times \cdots \times \mathbb{R} P^{n_{k}}\right)=4 n-2 \alpha(n)+1 .
$$

\section{REFERENCES}

[1] G. E. Bredon. Topology and Geometry. Graduate Texts in Mathematics 139. Springer 1993. MR 1224675 (94d:55001)

[2] R. L. Cohen. The immersion conjecture for differentiable manifolds. Annals of Math. (2) (1985), 237-328. MR808220 (86m:57030)

[3] M. Ghomi, S. Tabachnikov. Totally skew embeddings of manifolds. Math. Z. 258 (2008), 499-512. MR 2369041 (2008m:57068)

[4] D. Handel, J. Segal. On $k$-regular embeddings of spaces in Euclidean space. Fund. Math. 106 (1980), 231-237. MR:584495 (81h:57005)

[5] J. Korbaš. Bounds for the cup-length of Poincaré spaces and their applications. Topology and its Applications 153, no. 15, 2006, 2976-2986. MR2248401(2007h:57031)

[6] W. S. Massey. On the Stiefel-Whitney classes of a manifold. Amer. J. of Math., 82 (1960), 92-102. MR0111053 (22:1918)

[7] J. W. Milnor, J. D. Stasheff. Characteristic Classes. Princeton Univ. Press, Princeton, NJ, 1974. MR0440554 (55:13428)

[8] G. Stojanović, S. Tabachnikov. Non-existence of $n$-dimensional $T$-embedded discs in $R^{2 n}$. Comment. Math. Helv. 81 (2006), 877-882. MR2271226 (2007i:53007)

[9] G. Stojanović. Embeddings with multiple regularity. Geom. Dedicata 123 (2006), 1-10. MR 2299723 (2008c:53005) 
[10] G. Stojanović. Embeddings with certain non-degeneracy conditions. Ph.D degree thesis. Pennsylvania State University, August 2007. MR2714236

[11] R. E. Stong. Cup products in Grassmannians. Topology Appl. 13 (1982), 103-113. MR637432 (83c:55003)

Mathematical Institute, Serbian Academy of Sciences \& Arts, Kneza Mihaila 36, P.P. 367, 11001 Belgrade, Serbia

E-mail address: djbaralic@mi.sanu.ac.rs

$U R L:$ http://www.sanu.ac.rs/English/Index.aspx

Faculty of Mathematics, University of Belgrade, Studentski Trg 16, 11000 BelGRADE, SERBIA

E-mail address: bane@matf.bg.ac.rs

Mathematical Institute, Serbian Academy of Sciences \& Arts, Kneza Mihaila 36, P.P. 367, 11001 Belgrade, Serbia

E-mail address: golence@gmail.com

Faculty of Mathematics, University of Belgrade, Studentski Trg 16, 11000 BelGRADE, SERBIA

E-mail address: vrecica@matf.bg.ac.rs

Mathematical Institute, Serbian Academy of Sciences \& Arts, Kneza Mihaila 36, P.P. 367, 11001 Belgrade, Serbia

E-mail address: rade@mi.sanu.ac.rs 